\title{
Entwicklung eines Demontagesystems für Waschmaschinen innerhalb eines Wertstoffkreislaufes
}

\author{
Bernd Hentschel, Thomas Kindler
}

\section{Einleirung}

Das Institut für Unternehmenslogistik am Technologietransfer- und Weiterbildungszentrum an der Technischen Fachhochschule Wildau ist im Rahmen des Logistikverbundes Berlin-Brandenburg gemeinsam mit innovativen Logistikdienstleistern stark in der anwendungsorientierten Projektarbeit integriert. So werden gemeinsam im Fachgebiet Logistik ganzheitliche Lösungskonzepte vom Entwurf bis zur Realisierung bearbeitet. Das gemeinsame Aufgabenspektrum reicht dabei von produktions- über entsorgungslogistische bis hin zu verkehrs- und vertriebslogistische Projekte. Als kombiniertes Beispiel dieser konkreten Projektarbeit zwischen Produktions- und Entsorgungslogistik wird nachfolgend die Entwicklung eines Demontageprozesses als Teil eines Wertstoffkreislaufes für das Altprodukt Waschmaschine mit der spezifischen Lösung des Demontageprozesses beschrieben.

Zwei Unternehmen aus den neuen Bundesländern - die amerikanische Firma O.K. Bley Engineering GmbH mit Sitz in Schönebeck/Sachsen-Anhalt und das Unternehmen RASOMA Werkzeugmaschinen $\mathrm{GmbH}$ mit Sitz in Döbeln/Sachsen beauftragten gemeinsam die Mitglieder des Logistikverbundes - Institut für Unternehmenslogistik und den Logistik- und Ingenieurdienstleister Projektlogistik GmbH - mit der grundsätzlichen Entwicklung eines logistischen Gesamtkonzeptes für ein Demontagesystem und der zugehörigen Materialflußlösung einschließlich des entsorgungslogistischen Wertstoffkreislaufes. Ziel dieses Projektes war somit die Entwicklung einer Prototyplösung für diese Unternehmen als innovative Demontagelösung und deren spezifischer Vermarktung durch die betreffenden Auftraggeber als potentielle Partner der maschinenbaulichen Komponente.

\section{Lösungsansatz}

Ausgehend von einer repräsentativen Anwendung dieser Demontagesysteme für das gesamte Spektrum der Braunen und Weißen Ware wurden unterschiedlichste Altprodukte nach Auftraggebervorgabe (Waschmaschinen, Kühlschränke bis hin zu Elektrozählern) manuell demontiert und die besonderen Schwierigkeiten und Probleme der Zerlegungsprozesse nach technologischen und verfahrenstechnischen Kriterien erfaßt.

Beispielgebend für die Waschmaschinendemontage wurden z. B. für vier verschiedene Waschmaschinentypen (Bauknecht WA 590/Frontlader, Hanseatic 1000 B
Frontlader, Bosch VT 585 Frontlader und FORON VA 561

Toplader) nach den Kriterien

- der automatisierbaren Demontageverrichtungen mit erforderlicher Genauigkeitsanforderung,

- der nicht oder nicht ökonomisch automatisierbaren Demontageverrichtungen,

- Ermittlung sinnvoller Fraktionierungsstufen im Demontageprozeß auf Basis geometrischer und verfahrenstechnischer Kriterien,

- den erzielbaren manuellen Demontagezeiten,

- den wahrscheinlich erzielbaren Demontagezeiten im automatisierten Betrieb.

- den erforderlichen Demontagewerkzeugen mit Definition von Neuentwicklungen im automatisierten und manuellen Betrieb und erforderlicher Werkzeugwechselzeiten,

- den Handhabemassen und geometrischen Greifbedingungen sowie

- den erforderlichen Hilfs-, Halte- und Unterstützungskonstruktionen mit erforderlicher Prozeßzeitbeanspruchung für manuellen und automatisierten Betrieb untersucht.

Aus verschiedenartigsten Untersuchungen in der Literatur und bereits gesammelter Erfahrungen in der Entwicklung von Demontagesystemen wurde über den Baugruppengraph bereits die Entscheidung zu automatisierbarer bzw. nur manueller Demontage abgeleitet. In den nachfolgenden Abbildungen sind dazu je ein Auszug aus dem Baugruppengraph sowie den empirisch ermittelten Demontagetechnologien je Demontageverrichtung dargestellt.

Mit diesen außerordentlich umfangreichen Ist-Ermittlungen der Demontageerfordernisse war somit die Möglichkeit gegeben, die notwendige Grobstruktur des zu konzipierenden Systems deutlich zu umreißen. Es zeigte sich, daß für die automatisierte Demontage der Trommelausbau, die Gehäusedemontage, die Kabelbaumdemontage möglich sind und für den manuellen Demontagebereich Bedienelemente, Abdeckplatte und Beladungsplatte sowie die weitere Trommeldemontage als auch die restliche Gehäusedemontage infrage kommen. Eine andere und größere Demontage- und Fraktionierungstiefe erschien aus den Untersuchungen nicht sinnvoll. Damit zeigte sich auch hier die bereits gemachte Erfahrung, dieses System als kombiniertes System einer gemischt manuellen und automatisierten Variante für den Auftraggeber zu entwickeln.

Die Anforderungen sind derart zu beschreiben, daß das gesamte System für Positionierung und systeminternen 
Typ Bauknecht WA 590

Baugruppengraph

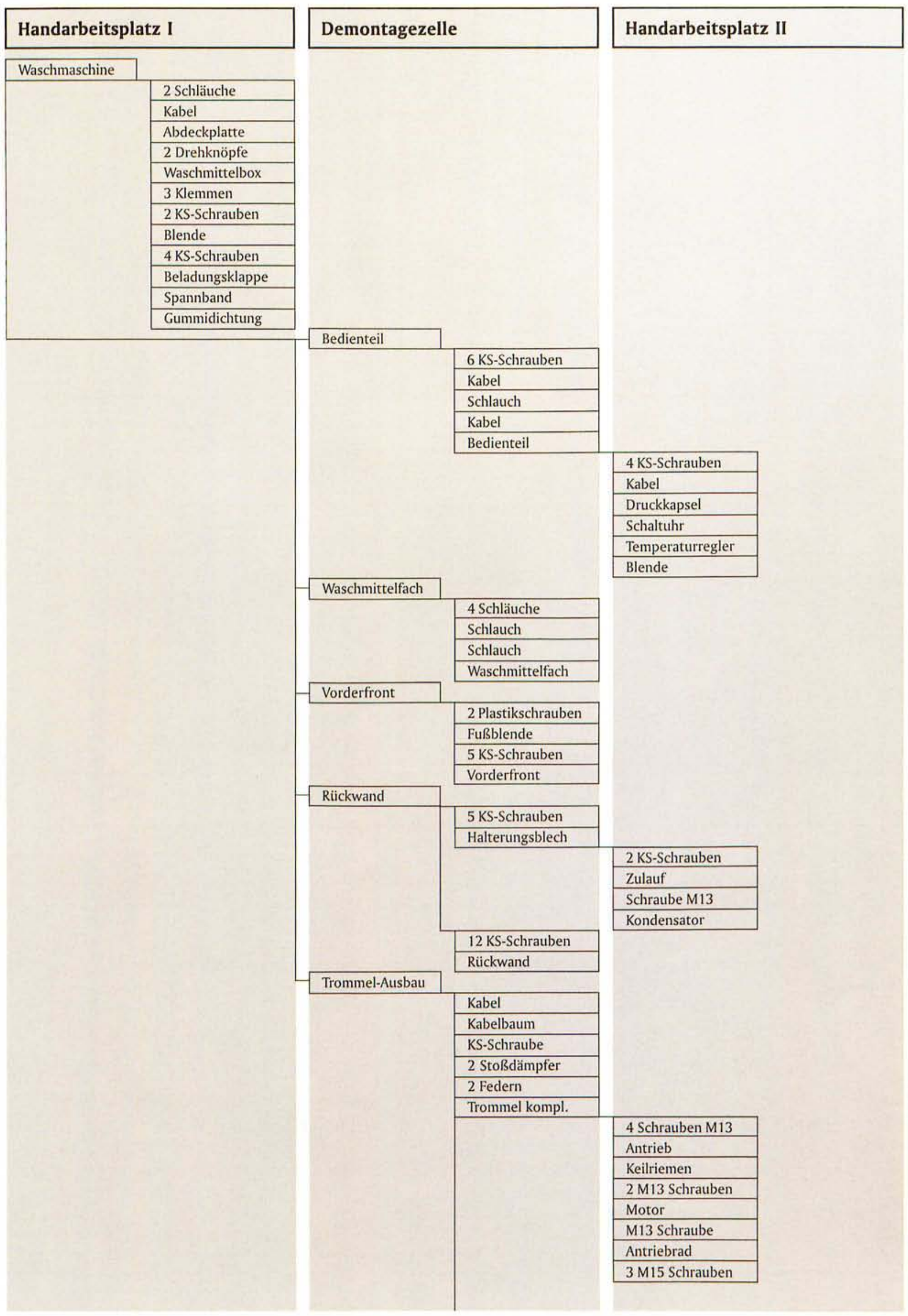




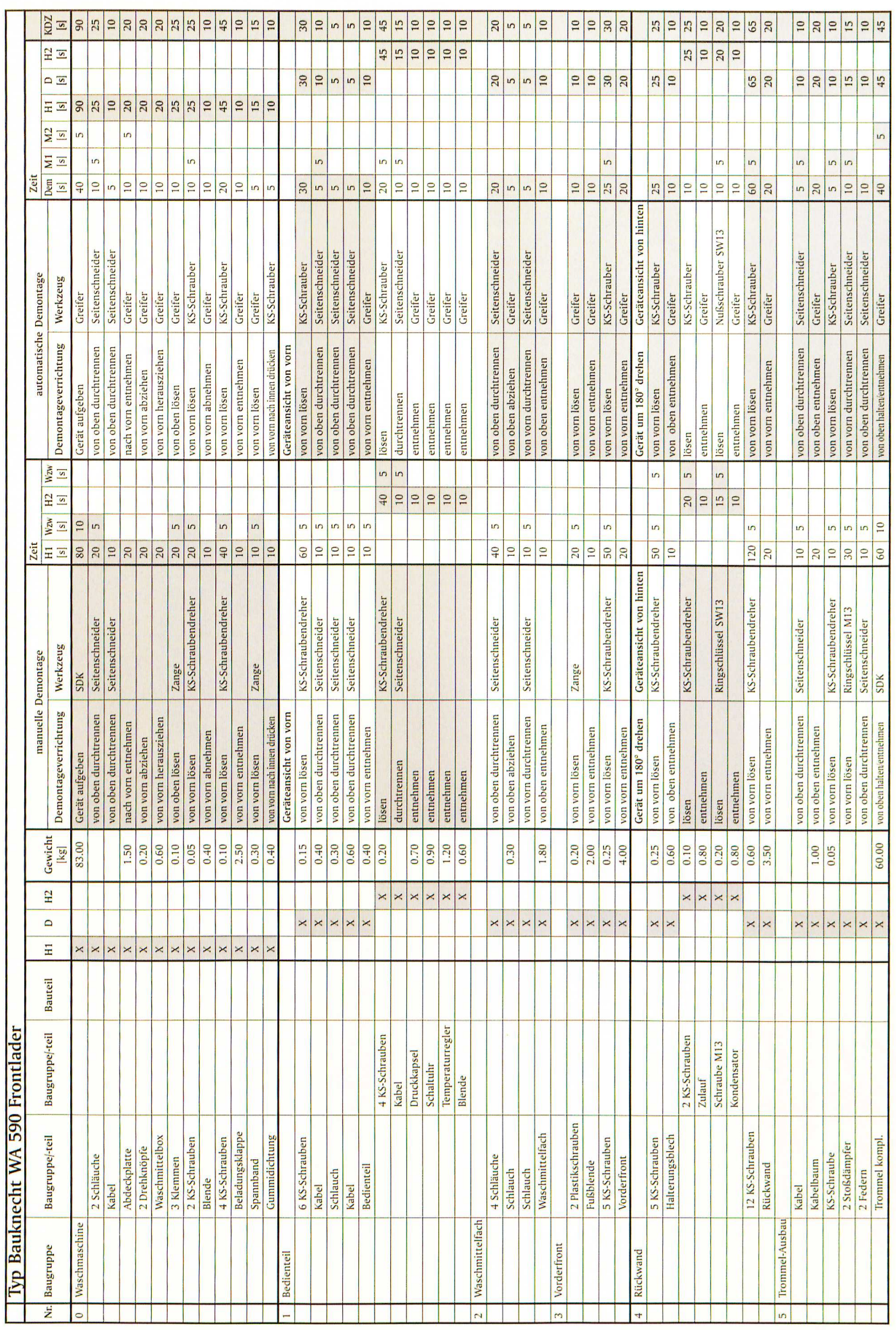

(Abkiirzungen: D - Demontagezelle; H1/H2 - Handarbeitsplatz 1 und 2; M1/M2 - Manipulator 1 und 2; Dem - Demontagezeit; KDZ - Kombinierte Demontagezeit; Wzw - Werkzeugwechselzeit) 
Transport für eine maximale Masse von $60 \mathrm{~kg}$ auszulegen ist, die erforderliche Handhabetechnik für die automatisierte Demontage mit mindestens 6 Freiheitsgraden ausgelegt sein muß, ein erheblicher Vorrichtungsund Halteeinrichtungsbedarf sowie Werkzeugsonderbedarf mit kürzestmöglichen Werkzeugwechselzeiten im Bereich von $20-30$ s gesichert sein muß. Hinzu kommt eine integrierende Systemsteuerung mit Anbindung an die interne Unternehmenslogistik bezogen auf die Anlieferung der zu demontierenden Altgeräte sowie einer externen Ausschleus- und Lagerstrategie zur Deponierung nutzbarer Teile/Baugruppen bzw. Weiterleitung in den Aufbereitungs- und Regenerierungsteil des Wertstoffkreislaufes.

\section{Demontagesystemlösungen}

Im Rahmen eines morphologischen Vorgehens bei der Entwicklung verschiedenartigster Demontagesystemvarianten sollen stellvertretend für die Vielzahl dieser Möglichkeiten zwei Entwürfe stehen. Es bieten sich einerseits für das kombinierte manuell/automatisert betreibbare Demontagesystem im automatisiert arbeitenden Zyklus überflurgeführte Handhabetechnik mit kompletter Übernahme der erforderlichen Demontageverrichtungen an. Andererseits ist auch die Kombination flurgebundener mit überflurgeführter Handhabetechnik denkbar oder der reine Einsatz flurgebunder Knickarmrobotertechnik. So stehen die Abbildungen 3 und 4 in Form der Varianten 2 und 3 für diese Beispiele.

Allen Beispielen ist eigen, daß die Altprodukteaufgabe über eine Einschleusstation manuell mit Säulendrehkran und erforderlichen Werkstiickträgern erfolgt. Anschließend erfolgt eine manuelle Vordemontage mit folgender Geräteidentifikationsstelle zur richtigen Zuordnung der mittels Robotertechnik benötigten automatisierten Demontagetechnologie. Diese Technologien werden über die manuelle Demontage ermittelt und als Datei im Systemrechner hinterlegt. Die systeminterne Transporttechnik wird über angetriebene Rollenförderer bzw. in den nachfolgenden Transportprozessen über Bandförderer realisiert. Die nachfolgenden Arbeitsplätze werden durch den Portalroboter in Linienausführung (Querund Längsanordnung) bestimmt und enden entweder in Ablagebändern für demontierte Teile/Baugruppen bzw. in manuellen Handdemontageplätzen mit wieder dort angebrachter Ablage oder interner Transportstrecke zum Weiterleiten demontierter Elemente. Nicht wiederverwendbare oder wiederaufbereitbare Teile/Elemente werden prozeßintern über ein integriertes Transportsystem zu einer separaten Shredderanlage transportiert. Mit diesen Systemlösungen wird gesichert, daß in relativ kompakter Weise Demontagesysteme mit höchster Innovation zum Einsatz gelangen können.

Aus der Variantenvielfalt wurde letztlich eine Lösung entsprechend Abbildung 5 gemeinsam mit den Auftraggebern fixiert. Sie wird als Reihenlösung im Materialfluß unter Nutzung von zwei flurgebundenen Knickarmrobotern in Kombination mit manueller Demontage genutzt. Über die bekannte Einschleusstation erfolgt der systeminterne Transport ohne Werkstückträger auf spezifischen Förderbändern. Vor dem automatisierten Demontagearbeitsplatz ist eine erste manuelle Vordemontage erforderlich. Hier müssen bestimmte Bau-

\begin{tabular}{|lc|}
\hline $\begin{array}{l}\text { O.K. BLEY } \\
\text { ENGINEERING GmbH }\end{array}$ & RASOMA \\
\hline
\end{tabular}

\section{Automatisierte Demontage von Waschmaschinen - Variante 2}

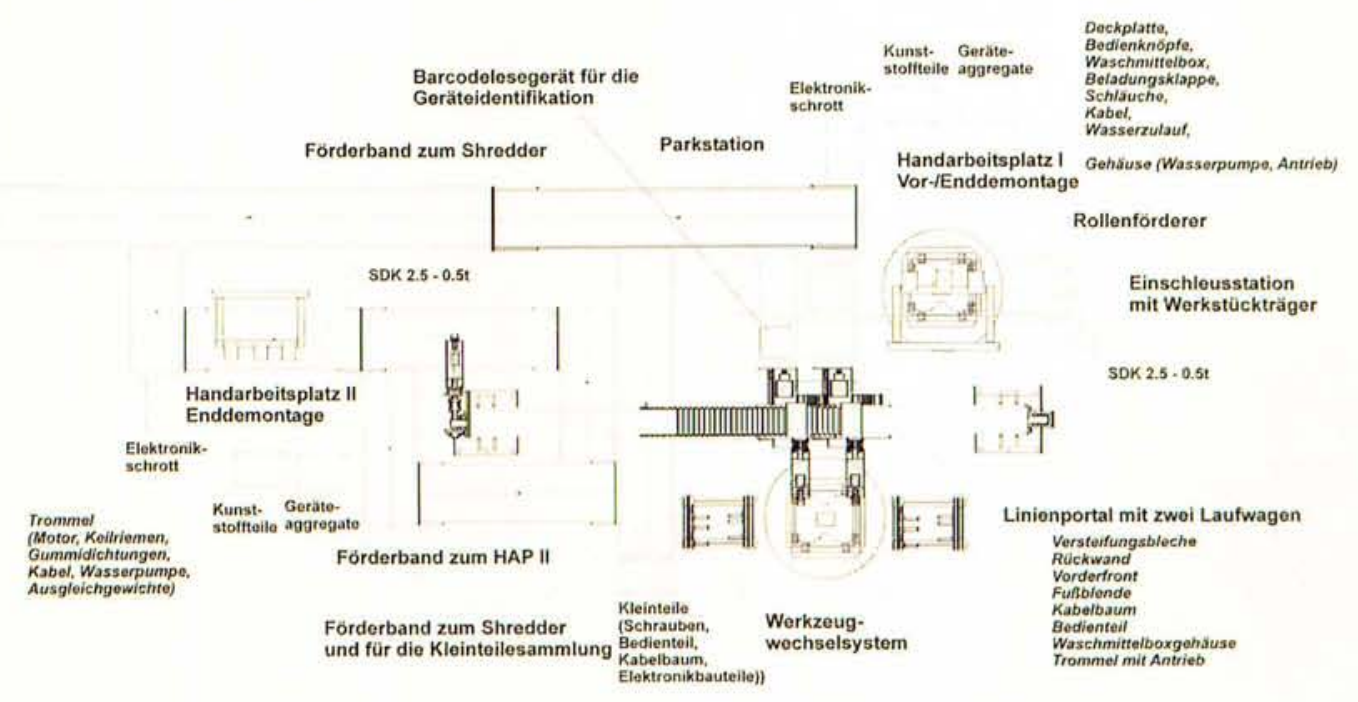

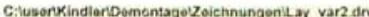

Bild 3: Automatisierte Demontage von Waschmaschinen in Linienportalausführung überflur - Variante 2 


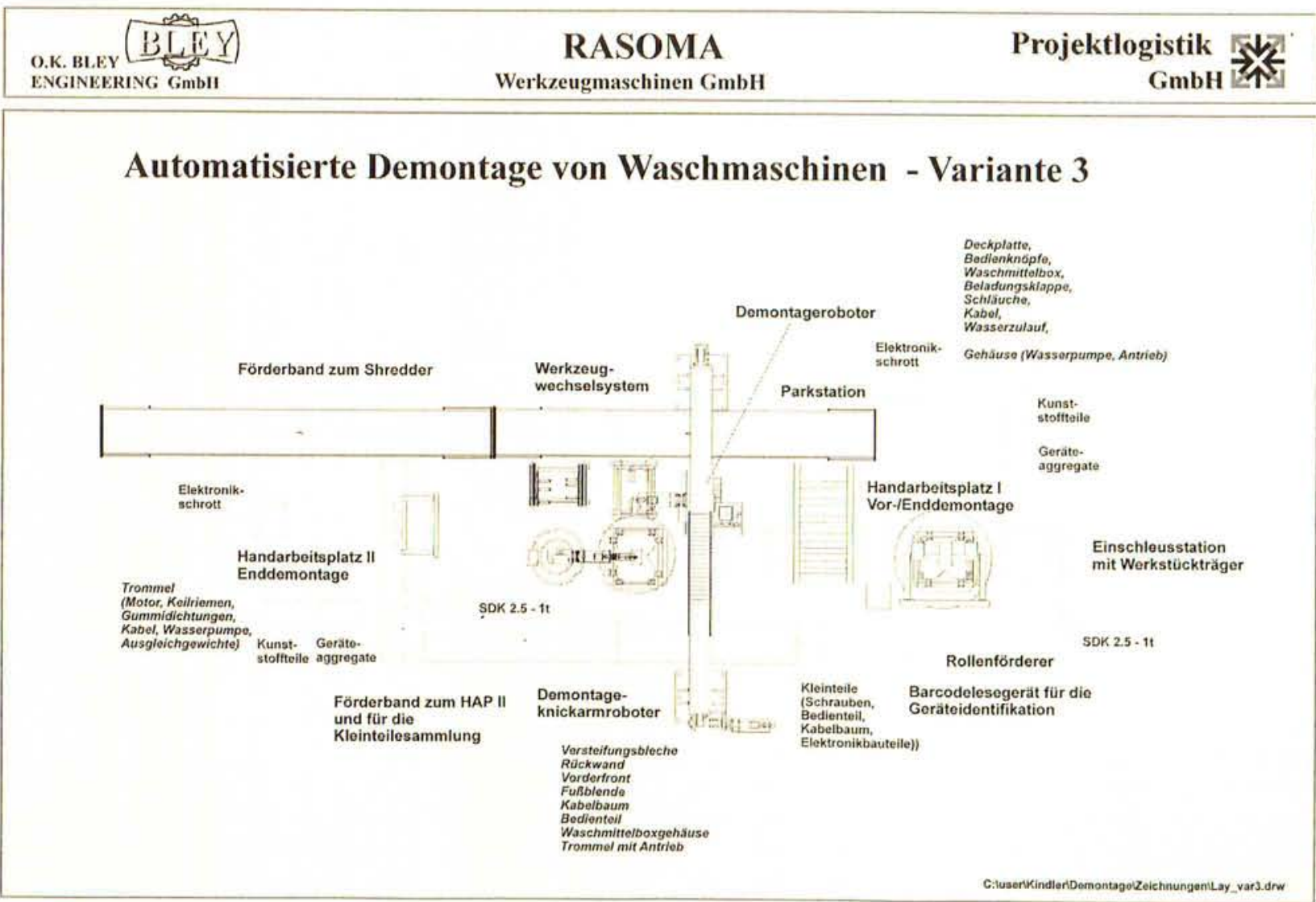

Bild 4: Automatisierte Demontage von Waschmaschinen in Linienportalausführung überflur und flurgebundenem Knickarmroboter - Variante 3

\begin{tabular}{|c|c|c|}
\hline BLEY & $\begin{array}{c}\text { RASOMA } \\
\text { Werkzeugmaschinen GmbH }\end{array}$ & $\underset{\text { PmbH }}{\text { Projektlogistik }}$ \\
\hline
\end{tabular}

\section{Automatisierte Demontage von Waschmaschinen - Variante 4}

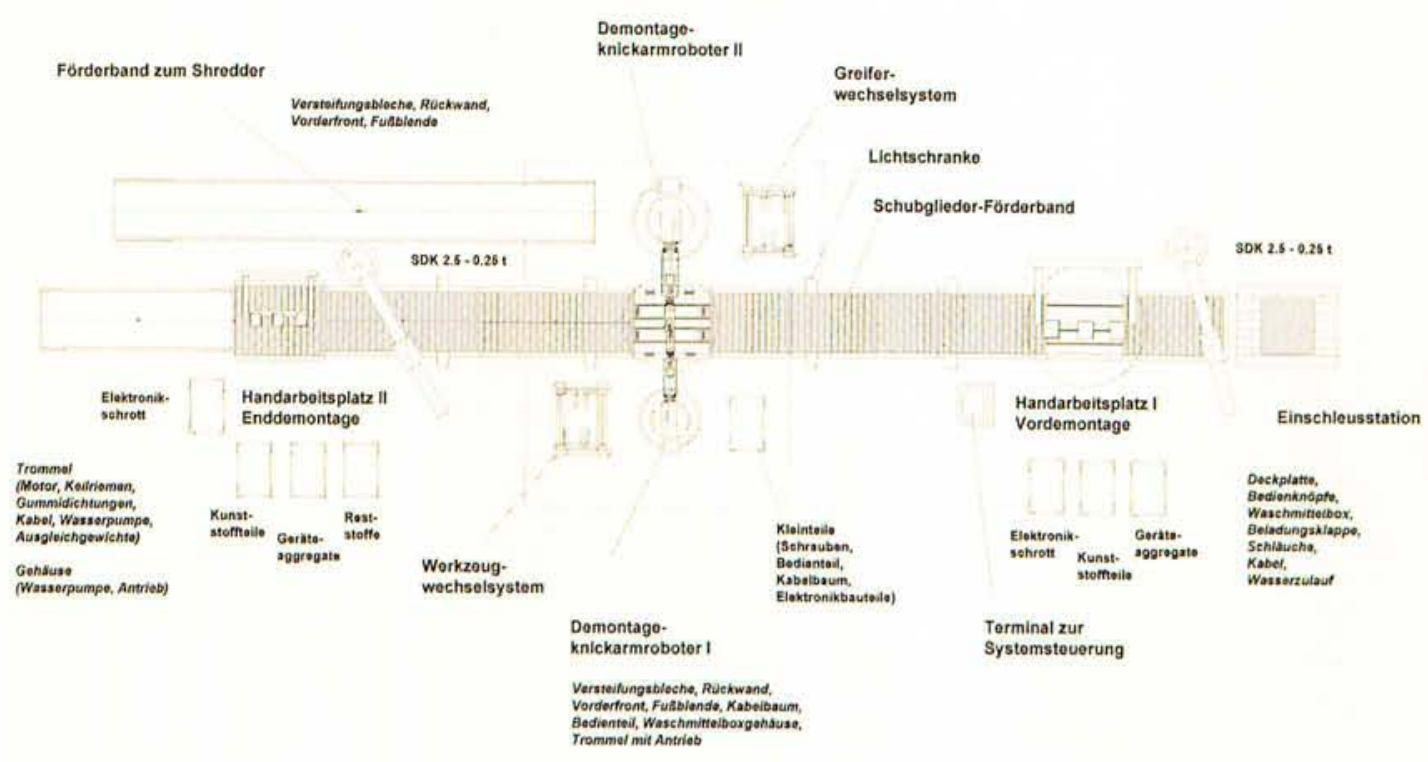

Bild 5: Systemgestaltung der Vorzugslösung Automatisierte Demontage von Waschmaschinen 


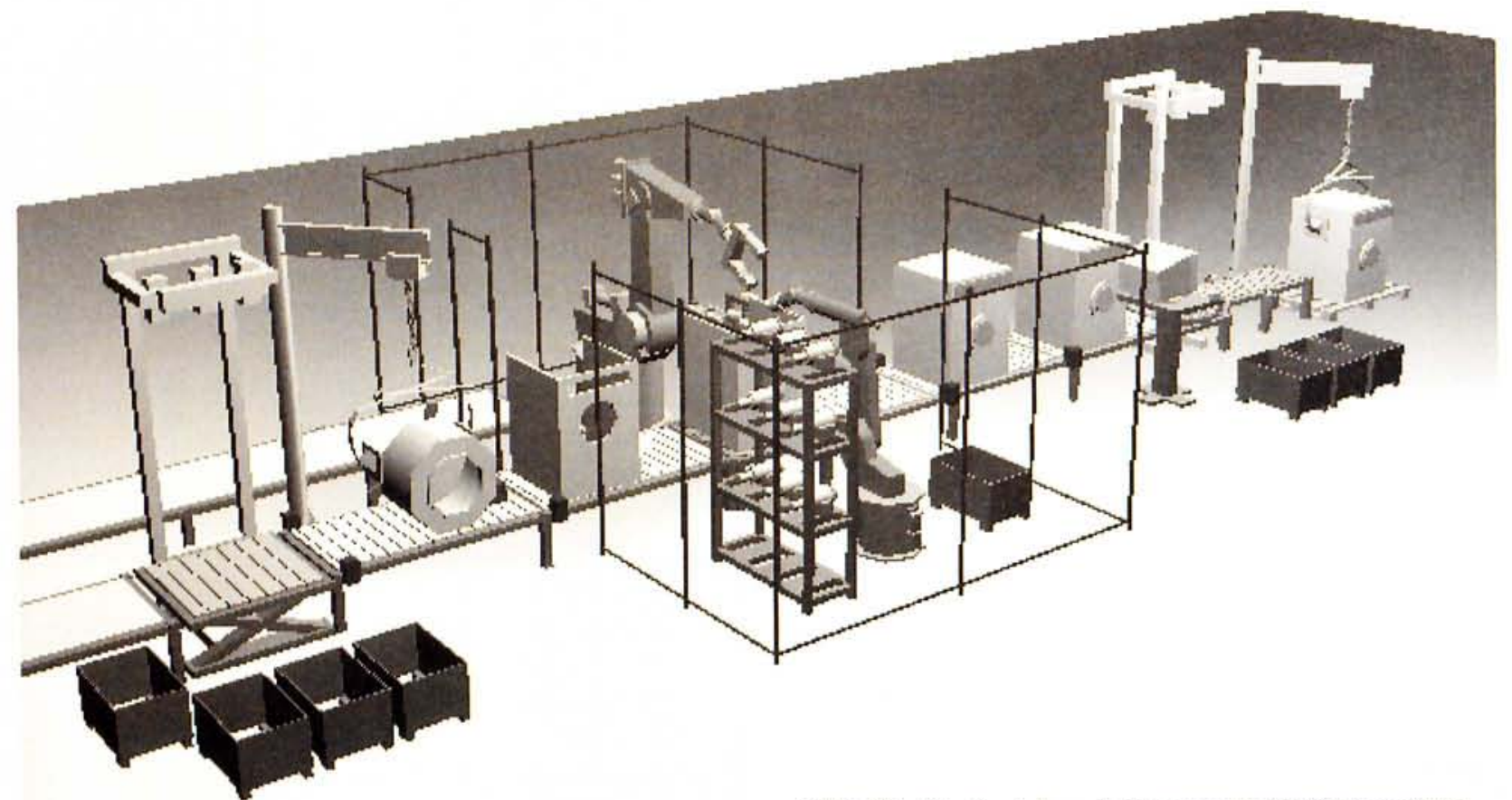

Bild 6: Räumliche Darstellung des Demontagemoduls Waschmaschinen

gruppen manuell demontiert werden, die ansonsten unökonomisch im automatisierten Zyklus abgebaut werden. Der automatisierte Demontagebereich ist selber als eigenständige Zelle mit integrierter Werkzeugwechsel- und Hilfseinrichtungsstation ausgerüstet. Die nachfolgenden Arbeitsplätze sind wiederum manueller Art und beinhalten die Demontage derzeit nicht ökonomisch handhabbarer Teile/Baugruppen bis zu einer definierten Fraktionierungsstufe. Die Ausschleusung wertloser Elemente erfolgt entweder je nach technologischer Folge über die Knickarmroboter oder aus den nachgelagerten Handarbeitsplätzen auf Transportbänder für den folgenden Shredderarbeitsgang. Die gesamte Lösung als räumliches Modell zeigt Abbildung 6 .

Selbstverständlich sind zum Betreiben dieser Lösung weitere Entwicklungsaufwendungen notwendig gewesen. Allein das gesamte Spektrum der Spezialwerkzeuge einschließlich des Werkzeugwechselsystems mit bis zu 10 integrierten Werkzeugen je Roboter und der erforderlichen Positionier- und Haltetechnik erforderten ein gleich großen Projektaufwand wie die Systemgrundlösung. Festzuhalten ist an dieser Stelle, daß diese Entwicklungen mit größeren Innovationsbestandteilen versehen waren und der besonderen Schwierigkeiten der Demontageprozesse angepaßt werden mußten.

Nicht verschwiegen werden darf in diesem Zusammenhang die ökonomische Betrachtung im Sinne der erforderlichen Entwicklungs- und Investitionskosten im Verhältnis zur Erlössituation. Derzeit ist mit dieser Lösung noch kein überzeugendes ökonomisches Arbeiten gesichert und es bedarf weiterer Arbeiten in Entwicklung und Logistik um für den potentiellen Anwender die sinnvolle Anwendung definieren zu können.

Andererseits ist jedoch festzuhalten, daß bei Inkrafttreten einer durchgehenden Rücknahmeverpflichtung je Hersteller und einer weltweit arbeitenden internetorientierten Vertriebslösung mit zertifizierten wiederaufbereiteten Teilen und Baugruppen mit zugeordneter Restlebensdauer die ökonomisch sinnvolle Barriere überschritten wird. Darauf sind alle weiteren Anstrengungen verschiedenartigster Entwickler in Demontagebereichen und Logistik bereits heute ausgerichtet.

\section{Ausblick und Zusammenfassung}

Gemeinsam mit den auftraggebenden Unternehmen und über eine durch die T.IN.A. Brandenburg GmbH eröffnete Recyclinginitiative Brandenburg wird unter Führung der Technischen Fachhochschule Wildau und dem Institut für Unternehmenslogistik an einer Variante gearbeitet, dieses System in modularer Form für unterschiedlichste Altprodukte im Europäischen Transport- und Logistikzentrum Preschen (Brandenburg) zu realisieren. In kooperativer Zusammenarbeit haben sich derzeit mehrere Unternehmen zusammengeschlossen und bereiten durch gezielte eigene Weiterentwicklungen in Verbindung mit der Technischen Fachhochschule Wildau diesen Anwendungsfall vor.

Ergänzt wird diese Weiterentwicklung durch die Erarbeitung einer Redistributionslösung sowie um die weltweite Vertriebslogistik inform der geprüften und mit einer Restlebensdauer versehenen wiederverwendbaren bzw. weiterverwendbaren Elemente/Teile und Baugruppen.

\section{Verfasser}

Prof. Dr.-Ing. habil. Bernd Hentschel

Dipl.-Ing. Thomas Kindler

Technische Fachhochschule Wildau

Fachbereich Ingenieurwesen/Wirtschaftsingenieurwesen Tel. (0 33 75) 508-927 